\title{
THE MULTIVARIATE BLACK \& SCHOLES MARKET: CONDITIONS FOR COMPLETENESS AND NO-ARBITRAGE
}

\author{
JAN DHAENE, ALEXANDER KUKUSH, AND DANIËL LINDERS
}

\begin{abstract}
Анотація. In order to price multivariate derivatives, there is need for a multivariate stock price model. To keep the simplicity and attractiveness of the one-dimensional Black \& Scholes model, one often considers a multivariate model where each individual stock follows a Black \& Scholes model, but the underlying Brownian motions might be correlated. Although the classical one-dimensional Black \& Scholes model is always arbitrage-free and complete, this statement does not hold true in a multivariate setting.

In this paper, we derive conditions under which the the multivariate Black \& Scholes model is arbitrage-free and complete.
\end{abstract}

\section{INTRODUCTION}

The origin of the one-dimensional Black \& Scholes model goes back to the early work of L. Bachelier in the beginning of the 20th century. His revolutionary idea of using stochastic processes to model the behavior of a stock over time was the start of a long tradition in developing stock price models; see Bachelier (1900). One of these models is proposed in Samuelson (1965). The popularity of this model is to a large extent influenced by the possibility to develop a sound theory of option pricing, which was first introduced in Black and Scholes (1973) and extended in Merton (1973). Although there is a variety of stochastic processes which might be more suitable for capturing the random fluctuations of a stock or index, the one-dimensional Black \& Scholes model still remains the benchmark model, mainly due to its simplicity and the disposal of an option pricing formula.

Various popular derivatives have a pay-off depending on several dependent assets. For example, a basket (or index) option has a pay-off which depends on a basket of stocks. Extrapolating the classical approach to higher dimensions asks for a multivariate Black \& Scholes model. It is shown that within this model, one can derive tight bounds for various exotic options; see e.g. Carmona and Durrleman (2006), Deelstra et al. (2004), Dhaene et al. (2002b) and Vanduffel et al. (2005) among others. In the aforementioned papers, the price of a multivariate derivative is determined as the discounted expectation under a risk-neutral probability measure. It is important to ensure that such a pricing measure exists and if so, in which situations it is unique. From Delbaen and Schachermayer (2008) it is known that the existence and uniqueness of the risk-neutral measure are closely related to the absence of arbitrage opportunities and the completeness of the market under consideration.

\footnotetext{
Надійшла .

2000 Mathematics Subject Classification. Primary: 91B24 ; Secondary: 60H10, 60J60, 60J65.

Ключові слова $і$ фрази. Black \& Scholes, multivariate asset price models, arbitrage-free, completeness, Brownian motion, risk-neutral probability measure.

KU Leuven, Leuven, Belgium.

Taras Shevchenko National University, Kyiv, Ukraine.

KU Leuven, Leuven, Belgium.
} 
In this paper we derive conditions for arbitrage and completeness for two popular multivariate versions of the Black \& Scholes model. Furthermore we discuss the existence and uniqueness of the risk-neutral pricing measure in both situations. We will also derive conditions under which the two models are equivalent. Related results about arbitrage and completeness can be found in Björk (1998), Karatzas and Shreve (1998), Musiela and Rutkowski (2005) and Shiryaev et al. (1995). To make the paper self contained, we will revisit some of the existing results and repeat the proofs.

The financial market that we consider throughout the paper is described in Section 2. In Section 3 we deal with the canonical form of the Black 8 Scholes model and give conditions under which it is arbitrage-free and complete. This stock price model describes each stock price by a linear combination of independent Brownian motions. Alternatively, one can describe each individual stock by a single Brownian motion, but these drivers might be correlated. In general, this multivariate Black \& Scholes model is not equivalent to the model considered in Section 3. In Section 4 we provide conditions under which this model is arbitrage-free and complete. In Section 5, we derive reasonable assumptions such that the multivariate Black \& Scholes model can be equivalently described by correlated Brownian motions or by a linear combination of independent Brownian motion. Furthermore, we show that this version of the Black \& Scholes model is always arbitrage-free and complete.

\section{The FinANCIAL MARKeT}

Consider the filtered probability space $\left(\Omega, \mathcal{F},\left(\mathcal{F}_{t}\right)_{0 \leq t \leq T}, \mathbb{P}\right)$ to describe a financial market consisting of $n$ traded stocks and a bank account. We assume that the filtered probability space satisfies the usual 'technical' conditions of completenss and rightcontinuity, and also that $\mathcal{F}_{0}$ contains all $\mathbb{P}$-null sets of $\Omega$ and all sets of $\mathbb{P}$-probability 1. Furthermore, we take $\mathcal{F}_{T}=\mathcal{F}$. Money can be deposited on or borrowed from the bank at the continuously compounded interest intensity $r \geq 0$, which is assumed to be deterministic and constant over time.

The current price of stock $i, i=1,2, \ldots, n$, is given by $X_{i}(0)$, which is assumed to be positive and nonrandom. The price of stock $i$ at future dates $t>0$ is described by the adapted stochastic process $\left\{X_{i}(t) \mid 0 \leq t \leq T\right\}$ which is defined on the above mentioned filtered probability space. Each stock $i$ pays a continuous dividend yield $\nu_{i}$, which is assumed to be deterministic and constant over time. Denoting the stochastic value of the (non-discounted) dividends paid in $(0, t)$ by $D_{i}(t)$, we have that

$$
\mathrm{d} D_{i}(t)=\nu_{i} X_{i}(t) \mathrm{d} t .
$$

The gains process $\left\{G_{i}(t) \mid 0 \leq t \leq T\right\}$ of stock $i$ is defined by

$$
G_{i}(t)=X_{i}(t)+\int_{0}^{t} \mathrm{e}^{r(t-u)} \mathrm{d} D_{i}(u), \quad t>0,
$$

with initial value $G_{i}(0)=X_{i}(0)$. If $\left\{X_{i}(t) \mid t \geq 0\right\}$ has continuous paths, then the integral in (2) is a path-wise Riemann integral, a.s.

Recall that a probability measure $\mathbb{Q}$ defined on $\left(\Omega, \mathcal{F},\left(\mathcal{F}_{t}\right)_{0 \leq t \leq T}\right)$ is said to be an equivalent martingale measure (or a risk-neutral measure) if $\mathbb{Q}$ is equivalent to $\mathbb{P}$ and any gains process $\left\{G_{i}(t) \mid 0 \leq t \leq T\right\}$, discounted at the risk-free rate, is a martingale with respect to $\mathbb{Q}$. In particular, under the risk-neutral measure $\mathbb{Q}$ we have that

$$
X_{i}(0)=\mathrm{e}^{-r t} \mathbb{E}\left[G_{i}(t)\right]=\mathrm{e}^{-r t} \mathbb{E}\left[X_{i}(t)\right]+\mathbb{E}\left[\int_{0}^{t} \mathrm{e}^{-r u} \mathrm{~d} D_{i}(u)\right] .
$$


In this expression and also hereafter, expectations have to be interpreted as expectations under the $\mathbb{Q}$-measure. Also, the notation $F_{X_{i}(t)}$ will be used to denote the cdf of the stock price $X_{i}(t)$ under $\mathbb{Q}$.

From the First Fundamental Theorem of Asset Pricing we know that the no-arbitrage condition is 'essentially equivalent' to the existence of an equivalent martingale measure $\mathbb{Q}$. For a detailed discussion of this fundamental theorem in mathematical finance, we refer to Delbaen and Schachermayer (2008). Consider a traded contingent claim $H$ which has a pay-off of $H(X(t))$ at time $t$. If the risk-neutral pricing measure in the market is given by $\mathbb{Q}$, then the price of $H(X(t))$ at time $s$, where $0 \leq s \leq t$, is equal to the conditional expected value of its discounted pay-off at time $t$ :

$$
\text { Price of } H \text { at time } s=\mathrm{e}^{-r(t-s)} \mathbb{E}\left[H(X(t)) \mid \mathcal{F}_{s}\right]
$$

where the expectation is taken with respect to $\mathbb{Q}$.

For each $i$, let us now construct a synthetic stock with stochastic price process $\left\{Y_{i}(t) \mid 0 \leq t \leq T\right\}$ defined by

$$
\frac{\mathrm{d} Y_{i}(t)}{Y_{i}(t)}=\frac{\mathrm{d} X_{i}(t)}{X_{i}(t)}+\nu_{i} \mathrm{~d} t, \quad t>0
$$

and with initial value given by $Y_{i}(0)=X_{i}(0)$. This means that

$$
Y_{i}(t)=X_{i}(0)+\int_{0}^{t} \frac{Y_{i}(u)}{X_{i}(u)} \mathrm{d} X_{i}(u)+\nu_{i} \int_{0}^{t} Y_{i}(u) \mathrm{d} u, \quad 0 \leq t \leq T,
$$

where the first integral on the right hand side is stochastic integral with respect to a diffusion process and the second one is Lebesgue integral. We can interprete $Y_{i}(t)$ as the value at time $t$ of an initial investment of amount $X_{i}(0)$ in stock $i$, where every dividend payment is immediately reinvested in stock $i$. Hence, $\left\{Y_{i}(t) \mid 0 \leq t \leq T\right\}$ can be interpreted as the price process of a non-dividend paying (synthetic) stock. From (4) we find for any $0 \leq s \leq t$ that

$$
Y_{i}(s)=\mathrm{e}^{-r(t-s)} \mathbb{E}\left[Y_{i}(t) \mid \mathcal{F}_{s}\right] .
$$

Taking into account (6), this expression can be transformed into

$$
X_{i}(s)=\mathrm{e}^{-\left(r-\nu_{i}\right)(t-s)} \mathbb{E}\left[X_{i}(t) \mid \mathcal{F}_{s}\right] .
$$

In particular, we have that

$$
X_{i}(0)=e^{-\left(r-\nu_{i}\right) t} \mathbb{E}\left[X_{i}(t)\right] .
$$

Comparing (3) and (8) leads to the following expression for the time-0 market price of the dividend payments in $(0, t)$ :

$$
\mathbb{E}\left[\int_{0}^{t} \mathrm{e}^{-r u} \mathrm{~d} D_{i}(u)\right]=X_{i}(0)\left[1-\mathrm{e}^{-\nu_{i} t}\right] .
$$

In the sequel of this paper, we always assume that each stock $X_{i}, i=1,2, \ldots, n$ pays no dividends in $[0, T]$. Our result remain valid in a more general context where some of the stocks are paying dividends, provided the earned dividends are reinvested in the stock.

2.1. Arbitrage-free and complete markets. Following Shiryaev et al. (1995), we define self-financing strategies and the notions of arbitrage and completeness. Loosely speaking, the financial market is said to be arbitrage-free if there is no trading strategy that does not require the injection of capital at initiation, while it leads to a non-negative gain with probability 1 and to a positive gain with a positive probability. The financial market is said to be complete if any contingent claim admits a replicating strategy. A 
precise definition of these notions requires a careful specification of the set of admissible investment strategies and admissible contingent claims.

Suppose that the total wealth of an investor at time $t$ is denoted by $G(t)$. This investor has an initial capital of $G(0)=x$ at time $t=0$. The available capital $G(0)$ is invested in a bank account $M$, with constant interest intensity $r$ and $M(t)=M(0) \mathrm{e}^{r t}$, and in the stocks $X_{1}, \ldots, X_{n}$ according to the portfolio

$$
\underline{\pi}=\left(\pi_{0}, \pi_{1}, \pi_{2}, \ldots \pi_{n}\right)^{T} .
$$

Here, we use the superscript ' $T$ ' to denote the transpose of a vector or matrix. Throughout the paper, all vectors have to be understood as column vectors.

The initial capital $G(0)$ can be written as

$$
G(0)=\pi_{0} M(0)+\pi_{1} X_{1}(0)+\pi_{2} X_{2}(0)+\ldots+\pi_{n} X_{n}(0) .
$$

In a similar way, let $\underline{\pi}(t)=\left(\pi_{0}(t), \pi_{1}(t), \pi_{2}(t), \ldots \pi_{n}(t)\right)^{T}$ be the $\mathcal{F}_{t}$-adapted collection of random variables describing a state of investor's securities portfolio at time $t>0$ :

$$
G(t)=\pi_{0}(t) M(t)+\pi_{1}(t) X_{1}(t)+\pi_{2}(t) X_{2}(t)+\ldots+\pi_{n}(t) X_{n}(t) .
$$

The vector process $(\underline{\pi}(t))_{t \geq 0}$ is called the investment strategy. Its components take any values in $(-\infty,+\infty)$. Following this investment strategy, the capital at time $t$ is denoted by $G_{\pi}(t)$. We consider only self-financing strategies $\pi$, i.e. such that for the capital (10) the integral representation

$$
G_{\pi}(t)=G_{\pi}(0)+\int_{0}^{t} \pi_{0}(u) \mathrm{d} M(u)+\int_{0}^{t} \pi_{1}(u) \mathrm{d} X_{1}(u)+\ldots+\int_{0}^{t} \pi_{n}(u) \mathrm{d} X_{n}(u)
$$

holds under the conditions ( $\mathbb{P}$-a.s., $t>0)$ :

$$
\int_{0}^{t}\left|\pi_{0}(u)\right| \mathrm{d} M(u)<\infty, \int_{0}^{t}\left(\pi_{i}(u) X_{i}(u)\right)^{2} \mathrm{~d} u<\infty, i=1,2, \ldots, n .
$$

The latter conditions provide the existence of the Lebesgue integral $\int_{0}^{t}\left|\pi_{0}(u)\right| \mathrm{d} M(u)$ $\mathbb{P}$-a.s. and of the stochastic integrals $\int_{0}^{t} \pi_{i}(u) \mathrm{d} X_{i}(u)$.

A self-financing strategy $\pi$ is called admissible at the time interval $[0, T]$ if there exists a non-random number $C>0$ such that

$$
\mathbb{P}\left[G_{\pi}(t) \geq-C, \text { for all } 0 \leq t \leq T\right]=1 .
$$

We denote the set of all such strategies by $S F_{a}$.

Definition 1 (Arbitrage-free market). The strategy $\pi \in S F_{a}$ is called an arbitrage on $[0, T]$ if $\mathbb{P}$-a.s.

$$
G_{\pi}(0) \leq 0, G_{\pi}(T) \geq 0
$$

and if

$$
\mathbb{P}\left[G_{\pi}(T)>0\right]>0 .
$$

If there are no arbitrage strategies in the market, then the market is called arbitrage-free.

Definition 2 (Complete market). The market is complete if for each $\mathcal{F}_{T}$-measurable payment function $f_{T}=f_{T}(\omega) \geq 0$, sucht that $\mathbb{E}\left[f_{T}^{1+\varepsilon}\right]<\infty$, for some $\varepsilon>0$, there exists a strategy $\pi \in S F_{a}$ such that $\mathbb{P}$-a.s.

$$
G_{\pi}(T)=f_{T} .
$$




\section{The multivariate Black \& Scholes model: Canonical form}

3.1. The real-world dynamics. Consider a market with $n$ non-dividend paying stocks, labeled from 1 to $n$. The price of stock $i$ at time $t$ is denoted by $X_{i}(t)$. We assume that the stock price dynamics can be described by the following set of stochastic differential equations (SDE's):

$$
\frac{\mathrm{d} X_{i}(t)}{X_{i}(t)}=\mu_{i} \mathrm{~d} t+\sum_{j=1}^{k} \bar{\sigma}_{i, j} \mathrm{~d} W_{j}(t), \text { for } t>0 \text { and } i=1,2, \ldots, n,
$$

where $\underline{W}(t)=\left(W_{1}(t), W_{2}(t), \ldots, W_{k}(t)\right)^{T}$ and $\{\underline{W}(t) \mid t \geq 0\}$ is a standard $k$-dimensional Brownian motion defined on the filtered probability space $(\Omega, \mathcal{F}, \mathbb{P})$. This probability space is equiped with the filtration $\left(\mathcal{F}_{t}^{W}\right)_{0 \leq t \leq T}$ of $\mathcal{F}$ which records the 'the past behavior' of the multivariate Brownian motion. This means that for $0 \leq t \leq T, \mathcal{F}_{t}^{W}$ denotes the $\sigma$ algebra generated by $\{\underline{W}(t) \mid 0 \leq s \leq t\}$, which we also denote by $\sigma\{\underline{W}(s) \mid 0 \leq s \leq t\}$, completed by events of zero probability $\mathbb{P}$. The vector $\underline{\mu}=\left(\mu_{1}, \mu_{2}, \ldots, \mu_{n}\right)^{T}$ contains the drift parameters of each stock and the matrix $\bar{\Sigma}$ is defined as

$$
\bar{\Sigma}=\left(\bar{\sigma}_{i, j}\right)_{i=1: n, j=1: k} \text {. }
$$

We have that $\mu \in \mathbb{R}^{n}, \bar{\sigma}_{i, j} \in \mathbb{R}$ and $\bar{\Sigma} \in \mathbb{R}^{n \times k}$.

Model (11) for the stock prices is called the canonical form of the multivariate Black 8 Scholes model.

3.2. Risk-Neutral dynamics. The quantity $\mu_{i}-r$ is called the risk-premium of stock $i$. It represents the excess return one receives in return for investing in stock $i$. In the Black \& Scholes model (11), the market consists of $k$ random sources and each stock is driven by a linear combination of these random sources. It is reasonable to assume that the contribution of each random source to the risk premium is the same for each stock. Otherwise stated, we should be able to find a vector $\underline{\gamma} \in \mathbb{R}^{k}$ satisfying the following condition:

$$
\mu_{i}-r=\sum_{j=1}^{k} \bar{\sigma}_{i, j} \gamma_{j}, \text { for } i=1,2, \ldots, n .
$$

The quantity $\gamma_{j}$ represents the effect on the risk-premium when the asset is exposed to one unit of volatility of the $j$-th risk driver $W_{j}$. If $k=n$ and $\bar{\Sigma}^{-1}$ exists, we have that $\underline{\gamma}$ is unique and equal to $\underline{\gamma}=\bar{\Sigma}^{-1}(\underline{\mu}-r \underline{1})$.

Define the $k$-dimensional process $\{\underline{\widetilde{W}}(t) \mid t \geq 0\}$ as follows:

$$
\begin{aligned}
& \mathrm{d} \widetilde{W}_{1}(t)=\mathrm{d} W_{1}(t)+\gamma_{1} \mathrm{~d} t, \\
& \mathrm{~d} \widetilde{W}_{2}(t)=\mathrm{d} W_{2}(t)+\gamma_{2} \mathrm{~d} t, \\
& \vdots \\
& \mathrm{d} \widetilde{W}_{k}(t)=\mathrm{d} W_{k}(t)+\gamma_{k} \mathrm{~d} t .
\end{aligned}
$$

It follows from Girsanov's theorem that we can find a probability measure $\mathbb{Q}$, equivalent to $\mathbb{P}$ and such that $\{\underline{\underline{W}}(t) \mid t \geq 0\}$ is a $k$-dimensional standard Brownian motion under this new probability measure. If (12) holds, plugging (12) into (11) and using relation (13) results in

$$
\frac{\mathrm{d} X_{i}(t)}{X_{i}(t)}=r \mathrm{~d} t+\sum_{j=1}^{k} \bar{\sigma}_{i, j} \mathrm{~d} \widetilde{W}_{j}(t), \text { for } i=1,2, \ldots, n
$$


Taking into account (14), it follows that $\{\underline{X}(t) \mid t \geq 0\}$ is an $n$-dimensional geometric Brownian motion under the probability measure $\mathbb{Q}$. Under this new probability measure, all the drifts are replaced by the risk-free rates while the parameters $\bar{\sigma}_{i, j}$ are remaining the same.

One can prove that in the $\mathbb{Q}$-world, the discounted price process of each stock is a martingale:

$$
\mathbb{E}_{\mathbb{Q}}\left[\mathrm{e}^{-r(t-u)} X_{i}(t) \mid \mathcal{F}_{u}\right]=X_{i}(u), \text { for } i=1,2, \ldots, n .
$$

The probability measure $\mathbb{Q}$ is equivalent to $\mathbb{P}$ and is called an equivalent martingale measure. The following theorem gives necessary and sufficient conditions for the multivariate Black \& Scholes model (11) to be arbitrage-free; see also Musiela and Rutkowski (2005), Karatzas and Shreve (1998) and Björk (1998).

Theorem 3 (Conditions for no arbitrage). In the multivariate Black 85 Scholes model (11), the following statements are equivalent:

(1) The model is arbitrage-free.

(2) There exists an equivalent martingale measure.

(3) There exists a non-random vector $\underline{\gamma}$ satisfying

$$
\mu_{i}=r+\sum_{j=1}^{k} \bar{\sigma}_{i, j} \gamma_{j}, \text { for } i=1,2, \ldots, n
$$

Доведення. $(3) \Longrightarrow(2)$

We already showed that if (16) holds, there exists a risk-neutral probability measure $\mathbb{Q}$.

$(2) \Longrightarrow(1)$ : The proof is standard; cf. Lemma 2 of Shiryaev et al. (1995).

$\Longrightarrow(3)$

A proof of this implication can also be found in Frey (2009). To make the paper selfcontained, we give a proof of this implication. What remains to be proven is that if (16) does not hold, we always have a market where there are arbitrage opportunities. A proof of this statement can be found in Karatzas and Shreve (1998). Here, we give a direct and straightforward proof to make this paper self-contained.

Assume that we cannot find a vector $\underline{\gamma}$ such that

$$
\mu_{i}=r+\sum_{j=1}^{k} \bar{\sigma}_{i, j} \gamma_{j}, \text { for } i=1,2, \ldots, n
$$

holds. Consider the portfolio $\underline{\pi}(t)=\left(\pi_{0}(t), \pi_{1}(t), \pi_{1}(t), \ldots, \pi_{n}(t)\right)^{T}$. The capital at time $t$ is denoted by $G^{\pi}(t)$ and can be expressed as

$$
G^{\pi}(t)=\pi_{0}(t) B(t)+\sum_{i=1}^{n} \pi_{i}(t) X_{i}(t),
$$

where $B(t)$ represents the riskless asset and $B(t)=\mathrm{e}^{r t}$. In the next lines, we prove that we can find an admissible, self-financing arbitrage strategy $\pi$, moreover we will have that

$$
G^{\pi}(0)=0, G^{\pi}(t)>0 \text {, a.s., for all } t>0 .
$$

The portfolio is assumed to be self-financing, hence

$$
\mathrm{e}^{r t} \mathrm{~d} \pi_{0}(t)+\sum_{i=1}^{n} X_{i}(t) \mathrm{d} \pi_{i}(t)=0 .
$$


Combining (18) and (19) results in

$$
\mathrm{d} G^{\pi}(t)=r G^{\pi} \mathrm{d} t+\sum_{i=1}^{n} \pi_{i}(t) X_{i}(t)\left(\left(\mu_{i}-r\right) \mathrm{d} t+\sum_{j=1}^{k} \bar{\sigma}_{i, j} \mathrm{~d} W_{j}(t)\right) .
$$

For the discounted capital $Y(t)=\mathrm{e}^{-r t} G^{\pi}$, we have by Ito's formula that

$$
\mathrm{d} Y(t)=\mathrm{e}^{-r t} \sum_{i=1}^{n} \pi_{i}(t) X_{i}(t)\left(\left(\mu_{i}-r\right) \mathrm{d} t+\sum_{j=1}^{k} \bar{\sigma}_{i, j} \mathrm{~d} W_{j}(t)\right) .
$$

There is an arbitrage opportunity if

or

$$
\sum_{j=1}^{k} \sum_{i=1}^{n} \pi_{i}(t) X_{i}(t) \bar{\sigma}_{i, j} \mathrm{~d} W_{j}(t) \equiv 0,
$$

$$
\sum_{i=1}^{n} \pi_{i}(t) X_{i}(t) \bar{\sigma}_{i, j} \equiv 0, \text { for all } j=1,2, \ldots, k .
$$

We denote by $f$ the vector $\left(\pi_{1}(t) X_{1}(t), \pi_{2}(t) X_{2}(t), \ldots, \pi_{n}(t) X_{n}(t)\right)^{T}$. Then $(21)$ is equivalent to $\bar{\Sigma}^{T} \underline{f}=0$ or $\underline{f} \in \operatorname{Ker}\left(\bar{\Sigma}^{T}\right)$. Note that we also have that $f \perp R(\bar{\Sigma})$. Because (17) does not hold, $\underline{\mu}-r \underline{1} \notin R(\bar{\Sigma})$. From the Fredholm Alternative, we find that there exists a vector $\underline{a}$ such that $\underline{a} \in \operatorname{Ker}\left(\bar{\Sigma}^{T}\right)$ and $\sum_{i=1}^{n} a_{i}\left(\mu_{i}-r\right) \neq 0$. We can set

$$
\underline{f}=\frac{\underline{a}}{\sum_{i=1}^{n} a_{i}\left(\mu_{i}-r\right)},
$$

so then $\underline{f} \in \operatorname{Ker}\left(\bar{\Sigma}^{T}\right)$. The vector $\underline{f}$ is a constant vector. We have that $\pi_{i}(t) X_{i}(t)=f_{i}$ and the strategy $\underline{\pi}(t)$ follows from $\pi_{i}(t)=\frac{f_{i}}{X_{i}(t)}$, for $i=1,2, \ldots, n$ and $\pi_{0}(t)$ can be found from (19).

For such trading strategy $\underline{\pi}(t)$, we find from (18) that

$$
\mathrm{e}^{r t} \mathrm{~d} Y(t)=\sum_{i=1}^{n} f_{i}\left(\mu_{i}-r\right) \mathrm{d} t
$$

We also have that $\sum_{i=1}^{n} f_{i}\left(\mu_{i}-r\right)=1$, so we can write $(22)$ as $\mathrm{e}^{r t} \mathrm{~d} Y(t)=\mathrm{d} t$, from which we find that

$$
Y(t)=\frac{1-\mathrm{e}^{-r t}}{r}>0, \text { for } t>0 .
$$

We can conclude that $G^{\pi}(t)>0$ for $t>0$, while $G^{\pi}(0)=0$. So the strategy $\underline{\pi}(t)$ is an arbitrage possibility, which proves that (16) should hold true if the market is assumed to be arbitrage-free.

A particular choice for the $\mathbb{Q}$-dynamics is to replace all the drift parameters $\mu_{i}$ by the risk-free rate $r$. Furthermore, each solution $\underline{\gamma}$ of (16) leads to an equivalent martingale measure.

The existence of a martingale measure is equivalent with stating that market (11) is arbitrage free. In the following theorem, we provide conditions under which the market is complete. We prove that the multivariate Black \& Scholes model (11) can be complete while there is no equivalent martingale measure.

Theorem 4 (Conditions for completeness). Consider the multivariate Black 83 Scholes model (11). Then the following statements are equivalent: 
(1) The market is complete.

(2) The variance-covariance matrix $\bar{\Sigma}$ satisfies

$$
\operatorname{rank}(\bar{\Sigma})=k .
$$

Доведення. (1) $\Leftrightarrow(2)$ : The sigma-algebra $\mathcal{F}_{t}^{X}$ denotes the filtration generated by the stock price process $\{\underline{X}(s) \mid 0 \leq s \leq t\}$. Assume now that $\operatorname{rank}(\bar{\Sigma})<k$, then it follows from (11) that

$$
\mathcal{F}_{t}^{X} \neq \mathcal{F}_{t}^{W}
$$

implying that the market is incomplete. Indeed the claim $f_{T}=\mathbb{I}(A)$ cannot be replicated by admissible strategies, where the event $A$ belongs to $\mathcal{F}_{t}^{W}$ and not to $\mathcal{F}_{t}^{X}$.

If $\operatorname{rank}(\bar{\Sigma})=k$, then we can form a submarket with $k$ stocks and non-singular variancecovariance matrix. It is proven in Karatzas and Shreve (1998) that such a market is complete. As a consequence, the market (11) is also complete because the filtrations in both situations coincide.

Condition (23) implies that $k \leq n$. To be complete, the number of random sources in the multivariate Black \& Scholes model (11) cannot exceed the number of traded stocks.

Corollary 1. Consider the multivariate Black 85 Scholes model (11). If the market is arbitrage-free, the following statements are equivalent:

(1) The market is complete.

(2) There exists a unique martingale measure.

\section{The multivariate Black \& Scholes model}

In this section we describe an alternative version of the multivariate Black \& Scholes model. Here, each stock $i$ will be modelled by a one-dimensional standard Brownian motion $B_{i}$, where the different Brownian motions are assumed to be dependent. Consider the financial market described in Section 2 and assume that the real world price dynamics are given by the following set of SDE's:

$$
\text { P-dynamics: } \frac{\mathrm{d} X_{i}(t)}{X_{i}(t)}=\mu_{i} \mathrm{~d} t+\sigma_{i} \mathrm{~d} B_{i}(t) \text {, for } t>0 \text { and } i=1,2, \ldots, n,
$$

where $\mu_{i} \geq 0$ is the drift and $\sigma_{i}>0$ the volatility of stock $i$. The parameters $\mu_{i}$ and $\sigma_{i}$ are assumed to be deterministic and constant over time.

Furthermore, $\left\{\left(B_{1}(t), B_{2}(t), \ldots, B_{n}(t)\right) \mid t \geq 0\right\}$ is an $n$-dimensional correlated Brownian motion process defined on the probability space $(\Omega, \mathcal{F}, \mathbb{P})$. This means that the stochastic processes $\left\{B_{i}(t) \mid t \geq 0\right\}$ are standard Brownian motions and that the dependence structure is captured by

$$
\sigma_{i, j} t=\operatorname{Cov}\left(\sigma_{i} B_{i}(t), \sigma_{j} B_{j}(t+s)\right),
$$

where $s \geq 0$. The process $\{\underline{B}(t) \mid t \geq 0\}$ is defined on a filtered probability space which is equiped with the filtration $\left(\mathcal{F}_{t}^{B}\right)_{0 \leq t \leq T}$ of $\mathcal{F}$. For $0 \leq t \leq T, \mathcal{F}_{t}^{B}$ is given by $\sigma\{\underline{B}(s) \mid 0 \leq s \leq t\}$, completed by events of zero probability $\mathbb{P}$.

The matrix $\underline{\Sigma}$ is given by $\underline{\Sigma}=\left(\sigma_{i, j}\right)_{i=1,2, \ldots, n ; j=1,2, \ldots, n}$ and we use $k$ to denote its rank. The correlation $\rho_{i, j}$ is for every $i, j=1,2, \ldots, n$ defined as

$$
\rho_{i, j}=\operatorname{Corr}\left(\sigma_{i} B_{i}(t), \sigma_{j} B_{j}(t+s)\right),
$$

and we can write that $\sigma_{i, j}=\rho_{i, j} \sigma_{i} \sigma_{j}$.

We prove that (24) can be rewritten in the canonical form (11). A related result can be found in Björk (1998). 
Theorem 5. Assume the dynamics of a multivariate stock price model are given by (24).

There always exists a matrix $\bar{\Sigma}=\left(\bar{\sigma}_{i, j}\right)_{i=1: n, j=1: k}$, with $\operatorname{rank}(\bar{\Sigma})=k \leq n$, such that the stock price dynamics can be described by

$$
\frac{\mathrm{d} X_{i}(t)}{X_{i}(t)}=\mu_{i} \mathrm{~d} t+\sum_{j=1}^{k} \bar{\sigma}_{i, j} \mathrm{~d} W_{j}(t), \text { for } i=1,2, \ldots, n,
$$

where $\{\underline{W}(t) \mid t \geq 0\}$ is a standard $k$-dimensional Brownian motion and the filtration $\mathcal{F}_{t}^{W}$ coincides with the filtration $\mathcal{F}_{t}^{B}$.

Доведення. For any $t>0$, define the vector $\xi(t)$ as

$$
\underline{\xi}(t)=\left(\sigma_{1} B_{1}(t), \sigma_{2} B_{2}(t), \ldots, \sigma_{n} B_{n}(t)\right)^{T},
$$

whereas $\xi(0)=0$. The orthogonal unit eigenvectors and eigenvalues of the matrix $\underline{\Sigma}$ are denoted by $\underline{e}_{i}$ and $\lambda_{i}$, respectively. Because the matrix $\underline{\Sigma}$ is a non-negative semidefinite matrix, all eigenvalues are positive. The rank of $\underline{\Sigma}$ is denoted by $k$. If $\lambda_{1} \geq \lambda_{2} \geq \ldots \geq \lambda_{n}$, we have that the first $k$ eigenvalues are strictly positive and $\lambda_{k+1}=\lambda_{k+2}=\ldots=\lambda_{n}=0$.

The vector $\underline{W}(t)=\left(W_{1}(t), W_{2}(t), \ldots, W_{k}(t)\right)^{T}$ is defined as

$$
W_{j}(t)=\frac{1}{\sqrt{\lambda_{j}}}\left\langle\underline{\xi}(t), \underline{e}_{j}\right\rangle, \text { for } j=1,2, \ldots, k,
$$

where $\langle.,$.$\rangle denotes the scalar product. It can be proven that \underline{W}(t)$ has a $k$-dimensional standard normal distribution and $W_{j}(0)=0$. The process $\{\underline{W}(t) \mid t \geq 0\}$ is a $k$-dimensional standard Brownian motion with continuous sample paths

Let $\bar{\Sigma}$ be the $n \times k$ matrix of rank $k$ containing the elements $\bar{\sigma}_{i, j}$ :

$$
\bar{\Sigma}=\left[\sqrt{\lambda_{1}} \underline{e}_{1} ; \sqrt{\lambda_{2}} \underline{e}_{2} ; \ldots ; \sqrt{\lambda_{k}} \underline{e}_{k}\right] .
$$

Define the $n$-dimensional Brownian motion $\{\widetilde{B}(t) \mid t \geq 0\}$ as follows:

$$
\widetilde{B}_{i}(t)=\frac{1}{\sigma_{i}} \sum_{j=1}^{k} \bar{\sigma}_{i, j} W_{j}(t), \text { for } i=1,2, \ldots, n .
$$

We have

$$
\mathbb{E}\left[\|\underline{\xi}(t)-\bar{\Sigma} \underline{W}(t)\|^{2}\right]=\mathbb{E}\left[\sum_{i=k+1}^{n}\left\langle\underline{\xi}(t), \underline{e}_{i}\right\rangle^{2}\right]=\sum_{i=k+1}^{n} \lambda_{i}=0 .
$$

So we find that $\mathbb{E}\left[\|\underline{B}(t)-\underline{\widetilde{B}}(t)\|^{2}\right]=0$, which implies that

$$
\int_{0}^{+\infty} \mathbb{E}\left[\|\underline{B}(t)-\underline{\widetilde{B}}(t)\|^{2}\right] \mathrm{d} t=0 .
$$

Applying Fubini's theorem proves that $\int_{0}^{+\infty}\|\underline{B}(t)-\underline{\widetilde{B}}(t)\|^{2} \mathrm{~d} t=0$, almost surely and

$$
\mathbb{P}[\underline{B}(t)=\underline{\widetilde{B}}(t), \text { for a.e. } t \geq 0]=1 .
$$

Both $\{\underline{B}(t) \mid t \geq 0\}$ and $\{\underline{\widetilde{B}}(t) \mid t \geq 0\}$ have continuous paths, from which we can conclude that

$$
B_{i}(t)=\frac{1}{\sigma_{i}} \sum_{j=1}^{k} \bar{\sigma}_{i, j} W_{j}(t), \text { for } i=1,2, \ldots, n,
$$

holds $\mathbb{P}$-a.s. This proves (26).

The two filtrations coincide because $\{\underline{B}(t) \mid t \geq 0\}$ and $\{\underline{W}(t) \mid t \geq 0\}$ are linear transformations of each other. 
Assume that the dynamics can be described by the canonical form (11) of the Black \& Scholes model. Then it does not hold true that we can rewrite these dynamics in the form of (24). Indeed, when $k>n$, it is not true that for each $t, \mathcal{F}_{t}^{W}=\mathcal{F}_{t}^{B}$.

The following theorem gives necessary and sufficient condition for the multivariate Black \& Scholes model (24) to be arbitrage-free and complete.

Theorem 6. The multivariate Black 8 Scholes model (24) is complete. For this model, the following statements are equivalent:

(1) The model is arbitrage-free.

(2) There exists an equivalent martingale measure.

(3) There exists a unique martingale measure $\mathbb{Q}$.

(4) There exists a non-random vector $\underline{\gamma}$ satisfying

$$
\mu_{i}=r+\sum_{j=1}^{k} \bar{\sigma}_{i, j} \gamma_{j}, \text { for } i=1,2, \ldots, n
$$

Доведення. We first write the dynamics (24) in the canonical form using Theorem 5 . It follows from Theorem 4 that the multivariate Black \& Scholes model (24) is complete.

The equivalence relations $(1) \Leftrightarrow(2) \Leftrightarrow(3)$ follow from Theorem 3 .

The proof of $(3) \Rightarrow(2)$ is trivial.

For a proof of $(2) \Rightarrow(3)$, assume that there are two equivalent martingale measures, which we denote by $\mathbb{Q}$ and $\mathbb{Q}^{*}$. Consider the event $A \in \mathcal{F}$ be any random event. Because the market is complete, the contingent claim $f_{T}=\mathbb{I}(A)$ can be replicated by the corresponding admissible self-financing strategy $(\underline{\pi}(t))_{t>0}$. The discounted capital $Y_{\pi}(t)$ is martingale under both $\mathbb{Q}$ and $\mathbb{Q}^{*}$ with underlying filtration. Then

$$
\mathrm{e}^{-r T} \mathbb{Q}(A)=\mathrm{e}^{-r T} \mathbb{E}_{\mathbb{Q}}[\mathbb{I}(A)]=Y_{\pi}(0),
$$

and in a similar way we find $\mathrm{e}^{-r T} \mathbb{Q}^{*}(A)=Y_{\pi}(0)$. We can conclude that $\mathbb{Q}(A)=\mathbb{Q}^{*}(A)$ for any $A \in \mathcal{F}$ which proves that $\mathbb{Q}=\mathbb{Q}^{*}$.

\section{An arbitrage-Free And COMPlete multivariate BlaCK \& SCholes model}

In this section we will show that under reasonable assumptions, we arrive at a special multivariate Black \& Scholes model for the stock price processes which is always arbitrage-free and complete.

In the canonical multivariate Black \& Scholes model (11), there are $k$ random sources for the $n$ traded stocks. We will first elaborate on the choice of $k$. More precisely, it will be shown that it is reasonable to assume that $k=n$ and $\operatorname{rank}(\bar{\Sigma})=n$. A first, intuitive, argument to avoid the situation where $k \neq n$ is the fact that it is not possible to construct a market where all the assets are independent when $k<n$ and if $k>n$, the market is not complete anymore.

5.1. $k>n$. Remember that $\bar{\Sigma}$ is an $n \times k$ matrix containing on row $i$ and column $j$ the parameter $\bar{\sigma}_{i, j}$. The rank of the matrix cannot exceed $n$. Assume for the moment that

$$
\operatorname{rank}(\bar{\Sigma})=n \text {. }
$$

This means that all the rows are linearly independent, while there are $(k-n)$ columns which can be expressed as linear combinations (l.c.) of the remaining $n$ columns. Without loss of generality, we may assume that the first $n$ columns are linearly independent, so each column $j=n+1, n+2, \ldots, k$ can be expressed as a l.c. of the first $n$ columns. To 
be more precise, for each $j=n+1, n+2, \ldots, k$, there exists a vector $\underline{\pi}^{(j)} \in \mathbb{R}^{n}$ such that

$$
\bar{\sigma}_{i, j}=\sum_{l=1}^{n} \pi_{l}^{(j)} \bar{\sigma}_{i, l}, \text { for } i=1,2, \ldots, n .
$$

Using this relation in (11) and changing the order of summation leads to

$$
\frac{\mathrm{d} X_{i}(t)}{X_{i}(t)}=\mu_{i} \mathrm{~d} t+\sum_{j=1}^{n} \bar{\sigma}_{i, j} \mathrm{~d} W_{j}(t)+\sum_{l=1}^{n} \bar{\sigma}_{i, l} \underbrace{\sum_{j^{\prime}=n+1}^{k} \pi_{l}^{\left(j^{\prime}\right)} \mathrm{d} W_{j^{\prime}}(t)}_{=\mathrm{d} M_{l}(t)} .
$$

The $n$-dimensional stochastic process $\{\underline{M}(t) \mid t \geq 0\}$ is defined as $M_{i}(t)=\sum_{j^{\prime}=n+1}^{k} \pi_{i}^{\left(j^{\prime}\right)} \mathrm{d} W_{j^{\prime}}(t)$, for $i=1,2, \ldots, n$. We can now rewrite the multivariate price dynamics (11) as

$$
\frac{\mathrm{d} X_{i}(t)}{X_{i}(t)}=\mu_{i} \mathrm{~d} t+\sum_{j=1}^{n} \bar{\sigma}_{i, j} \mathrm{~d}\left(W_{j}(t)+M_{j}(t)\right), \text { for } i=1,2, \ldots n .
$$

Although there are more than $n$ random sources, each stock can be described by a linear combination of $n$ 'funds', where each fund is a blend of the original random sources $W_{j}$, $j=1,2, \ldots, k$.

When $k>n$, the market is incomplete. Expression (28) shows that we can use a smaller number of financial instruments and change the filtration. As a result, the former incomplete market becomes complete.

5.2. $k<n$. Consider the $n \times k$ matrix $\bar{\Sigma}$ containing the parameter $\bar{\sigma}_{i, j}$ on row $i$ and column $j$. Assume that

$$
\operatorname{rank}(\bar{\Sigma})=k,
$$

so all the columns are linearly independent and it is not possible to reduce the $k$ random sources as we showed in Subsection 5.1. Because $k<n$, there are $(n-k)$ rows which can be expressed as linear combinations of the remaining $k$ rows. Without loss of generality we may assume that the first $k$ rows are linearly independent. For each $i=k+1, k+2, \ldots, n$, there exists a vector $\underline{\theta}^{(i)} \in \mathbb{R}^{k}$ such that

$$
\bar{\sigma}_{i, j}=\sum_{l=1}^{k} \theta_{l}^{(i)} \bar{\sigma}_{l, j}, \text { for } j=1,2, \ldots, k .
$$

For each $i=k+1, k+2, \ldots, n$ in (11), one obtains after changing the order of summation:

$$
\frac{\mathrm{d} X_{i}(t)}{X_{i}(t)}=\mu_{i} \mathrm{~d} t+\sum_{l=1}^{k} \theta_{l}^{(i)} \sum_{j=1}^{k} \bar{\sigma}_{l, j} \mathrm{~d} W_{j}(t) .
$$

Note that $\sum_{j=1}^{k} \bar{\sigma}_{l, j} \mathrm{~d} W_{j}(t)=\frac{\mathrm{d} X_{l}(t)}{X_{l}(t)}-\mu_{l} \mathrm{~d} t$, so we can write

$$
\frac{\mathrm{d} X_{i}(t)}{X_{i}(t)}=\mu_{i} \mathrm{~d} t+\sum_{l=1}^{k} \theta_{l}^{(i)}\left(\frac{\mathrm{d} X_{l}(t)}{X_{l}(t)}-\mu_{l} \mathrm{~d} t\right), \text { for } i=k+1, k+2, \ldots, n .
$$

Expression (29) shows that if $k<n$ and $\operatorname{rank}(\bar{\Sigma})=k$, only the first $k$ assets can be considered as primary assets. The remaining $(n-k)$ assets behave as funds which hold a portfolio consisting of the primary assets. 
5.3. An arbitrage-free and complete multivariate Black \& Scholes model. Given the discussion in Subsections 5.1 and 5.2, it is reasonable to assume that $k=n$ and $\operatorname{rank}(\bar{\Sigma})=n$. Under these assumptions, the reduction techniques explained in Subsections 5.1 and 5.2 cannot be applied and we are considering a market model which consists of $n$ primary assets which are driven by $n$ different random sources. In Theorem 7 , we prove that in this situation, the stock price dynamics can be equivalently described by $n$ correlated Brownian motions or by $n$ independent Brownian motions.

Theorem 7. The multivariate stock price dynamics can be equivalently described by the following two models.

(1)

$$
\frac{\mathrm{d} X_{i}(t)}{X_{i}(t)}=\mu_{i} \mathrm{~d} t+\sum_{j=1}^{n} \bar{\sigma}_{i, j} \mathrm{~d} W_{j}(t), \text { for } i=1,2, \ldots, n,
$$

where $\underline{\mu} \in \mathbb{R}^{n}$ and $\bar{\Sigma}=\left(\bar{\sigma}_{i, j}\right)_{i, j=1: n}$. The filtration at time $t \in[0, T]$ is $\mathcal{F}_{t}^{W}$ and $\operatorname{rank}(\bar{\Sigma})=n$.

(2)

$$
\frac{\mathrm{d} X_{i}(t)}{X_{i}(t)}=\mu_{i} \mathrm{~d} t+\sigma_{i} \mathrm{~d} B_{i}(t), \text { for } i=1,2, \ldots, n,
$$

where $\underline{\mu} \in \mathbb{R}^{n}$ and $\underline{\Sigma}=\left(\sigma_{i, j}\right)_{i, j=1: n}$. The dependence structure between the Brownian motions $B_{i}(t)$ is captured by (25). The filtration at time $t \in[0, T]$ is $\mathcal{F}_{t}^{B}$ and $\operatorname{rank}(\underline{\Sigma})=n$.

For each $t \in[0, T]$ we have that $\mathcal{F}_{t}^{W}=\mathcal{F}_{t}^{B}$ and $\underline{\Sigma}=\bar{\Sigma} \times \bar{\Sigma}^{T}$.

Доведення. From Theorem 5, we find in case the dynamics are described by model (31), we can always find a matrix $\bar{\Sigma}$, such that the dynamics are described by model (30), where the filtrations coincide.

Assume for the moment that the stock price dynamics are described by (30). For each $i$, we define $\sigma_{i}$ as $\sigma_{i}^{2}=\sum_{j=1}^{k} \bar{\sigma}_{i, j}^{2}$ and the stochastic process $\left\{B_{i}(t) \mid t \geq 0\right\}$ as

$$
B_{i}(t)=\frac{1}{\sigma_{i}} \sum_{j=1}^{n} \bar{\sigma}_{i, j} W_{j}(t) .
$$

It can be shown that $\left\{B_{i}(t) \mid t \geq 0\right\}$ is again a standard Brownian motion.

The process $\{\underline{B}(t) \mid t \geq 0\}$ is defined on a filtered probability space which is equiped with the filtration $\left(\mathcal{F}_{t}^{B}\right)_{0 \leq t \leq T}$ of $\mathcal{F}$. From (32), it follows that we can take $\mathcal{F}_{t}^{B}=\mathcal{F}_{t}^{W}$, for each $t \in[0, T]$. The quantity $\sigma_{i, j}$ is defined as

$$
\sigma_{i, j} t=\operatorname{Cov}\left(\sigma_{i} B_{i}(t), \sigma_{j} B_{j}(t+s)\right)
$$

and the variance-covariance matrix $\underline{\Sigma}$ is given by

$$
\underline{\Sigma}=\left(\begin{array}{cccc}
\sigma_{1}^{2} & \sigma_{1,2} & \cdots & \sigma_{1, n} \\
\sigma_{2,1} & \sigma_{2}^{2} & \cdots & \sigma_{2, n} \\
\vdots & \vdots & \ddots & \vdots \\
\sigma_{n, 1} & \sigma_{n, 2} & \cdots & \sigma_{n}^{2}
\end{array}\right),
$$

which shows that we can rewrite $(30)$ as $(31)$, where $\underline{\Sigma}=\bar{\Sigma} \times \bar{\Sigma}^{T}$.

A stock market where the different stocks are assumed to follow the multivariate Black \& Scholes model (30) or (31) is arbitrage-free and complete.

Theorem 8. The multivariate Black 85 Scholes models (30) and (31) are complete and arbitrage-free. 
Доведення. Both models are equivalent, so it suffices to prove that (30) is always arbitragefree and complete. Note that $\operatorname{rank}(\bar{\Sigma})=\operatorname{rank}(\underline{\Sigma})=n$. The matrix $\bar{\Sigma}$ is assumed to be of full rank, which implies that $\bar{\Sigma}^{-1}$ always exists. As a consequence, we find from Theorem 3 that the multivariate Black \& Scholes model (30) is always arbitrage-free. Indeed, we can always find a vector $\gamma \in \mathbb{R}^{n}$ such that (16) holds. Because $\operatorname{rank}(\bar{\Sigma})=n$, we find from Theorem 4 that (30) is a complete market.

\section{ACKNOWLEDGEMENTS}

The authors acknowledge the financial support of the Onderzoeksfonds KU Leuven (GOA/12/002/ TBA and GOA/13/002). They are grateful to Prof. Yu. Mishura and Dr. G. Shevchenko for fruitful discussions.

\section{Лiтература}

Bachelier, L. (1900), Theorie de la spéculation, PhD thesis, Faculté des sciences de Paris. Björk, T. (1998), Arbitrage theory in continuous time, Oxford University Press. pp. 311.

Black, F. and Scholes, M. (1973), 'The pricing of options and corporate liabilities', The Journal of Political Economy 81(3), pp. 637-654.

Carmona, R. and Durrleman, V. (2006), 'Generalizing the black-scholes formula to multivariate contingent claims', Journal of Computational Finance $\mathbf{9}$.

Deelstra, G., Liinev, J. and Vanmaele, M. (2004), 'Pricing of arithmetic basket options by conditioning', Insurance: Mathematics and Economics 34(1), $55-77$.

Delbaen, F. and Schachermayer, W. (2008), The mathematics of arbitrage, Springer. correcter second printing, pp. 373.

Dhaene, J., Denuit, M., Goovaerts, M., Kaas, R. and Vyncke, D. (2002b), 'The concept of comonotonicity in actuarial science and finance: applications', Insurance: Mathematics E) Economics 31(2), 133-161.

Frey, R. (2009), 'Financial mathematics in continuous time', University Lecture. statmath.wu.ac.at/ frey/Skript-FimaII.pdf.

Karatzas, I. and Shreve, S. E. (1998), Methods of Mathematical Finance, Springer-Verlag, New York.

Merton, R. C. (1973), 'Theory of rational option pricing', Bell Journal of Economics and Management 4(1), 141-183.

Musiela, M. and Rutkowski, M. (2005), Martingale methods in financial modeling, Springer-Verlag, Berlin. 2nd edition.

Samuelson, P. A. (1965), 'Proof that properly anticipated prices fluctuate randomly', Industrial Management Review 6, $41-49$.

Shiryaev, A. N., Kobanov, Y. M., Kramkov, O. D. and Melnikov, A. V. (1995), 'Toward the theory of pricing of options of both European and American types. ii. Continuous time', Theory of Probability and its Applications 39, 80 - 129.

Vanduffel, S., Hoedemakers, T. and Dhaene, J. (2005), 'Comparing approximations for risk measures of sums of non-independent lognormal random variables', North American Actuarial Journal 9(4), 71-82.

E-mail address: jan.dhaene@kuleuven.be

E-mail address: alexander_kukush@univ.kiev.ua

E-mail address: daniel.linders@kuleuven.be 